\title{
Abordaje laparoscópico en ruptura diafragmática traumática del lado derecho
}

\author{
Laparoscopic approach to right diaphragmatic rupture
}

\author{
Oscar Cervantes Gutiérrez, ${ }^{*}$ Caroline María de los Milagros Laroqcue Guzmán, * \\ Alberto Valdés Castañeda, ${ }^{*}$ Juan Pablo Arribas Martín, ${ }^{*}$ Carlos Mancera Steiner, ${ }^{*}$ \\ Alain Ledu Lara Calvillo, ${ }^{*}$ Marcos Jafif Cojab, ${ }^{*}$ Ana de la Cajiga León ${ }^{\ddagger}$ \\ * Departamento de Cirugía General. * Departamento de Anestesiología. \\ Hospital Ángeles Lomas. Ciudad de México, México.
}

\section{RESUMEN}

La ruptura diafragmática traumática es una entidad poco frecuente y de difícil diagnóstico dada la baja sospecha clínica y las lesiones que la acompañan. Presenta una incidencia de 1 a $7 \%$ en pacientes con traumatismo cerrado y de 10 a $15 \%$ en pacientes con traumatismos penetrantes. Se presenta el caso de un paciente con hernia diafragmática derecha por traumatismo contuso por accidente automovilístico, así como una revisión de la fisiopatología y las lesiones asociadas. Se hace especial énfasis en el diagnóstico radiológico y la importancia de la sospecha clínica. La tomografía computarizada es el estudio de referencia para el diagnóstico, la planeación quirúrgica y el tratamiento que involucra la reparación primaria del diafragma; se recomienda la vía laparoscópica. Se debe sospechar siempre esta patología para diagnosticarla a tiempo, especialmente en quienes han sufrido accidentes automovilísticos.

Palabras clave: Hernia diafragmática, trauma abdominal, trauma contuso, cirugía diafragmática, laparoscopía, toracoscopía.

\section{ABSTRACT}

Traumatic diaphragmatic rupture is a rare entity and difficult to diagnose given the low clinical suspicion and the accompanying injuries. It has an incidence of 1 to $7 \%$ in patients with blunt trauma and 10 to $15 \%$ in patients with penetrating trauma. The case of a patient with a right diaphragmatic hernia due to blunt trauma from a car accident is presented, as well as a review of the pathophysiology and associated injuries. Special emphasis is placed on radiological diagnosis and the importance of clinical suspicion. Computed tomography is the gold standard for diagnosis, surgical planning, and treatment involving primary diaphragm repair; the laparoscopic route is recommended. This pathology should always be suspected to diagnose it in time, especially in those who have suffered car accidents.

Keywords: Diaphragmatic hernia, abdominal trauma, blunt trauma, diaphragmatic surgery, laparoscopic, thoracoscopic.

\section{www.medigraphic.org. mx}

\author{
Recibido para publicación: 11/04/2020. Aceptado: 03/07/2020. \\ Correspondencia: Oscar Cervantes Gutiérrez. Departamento de Cirugía General, Hospital Ángeles Lomas, \\ Huixquilucan, Estado de México. Cel: 55 8794-0387. E-mail: ocervan@hotmail.com
}

Citar como: Cervantes GO, Laroqcue GCMM, Valdés CA, Arribas MJP, Mancera SC, Lara CAL et al. Abordaje laparoscópico en ruptura diafragmática traumática del lado derecho. Rev Mex Cir Endoscop. 2020; 21 (1): 36-40. https://dx.doi.org/10.35366/97612. 


\section{INTRODUCCIÓN}

La ruptura diafragmática traumática (TDR, por sus siglas en inglés) es poco común; tiene una incidencia que varía de 1 a $7 \%$ en pacientes con traumatismo contuso y de 10 a $15 \%$ en pacientes con traumatismos penetrantes. ${ }^{1}$ El lado de la ruptura diafragmática se correlaciona con la dirección del impacto, siendo el lado izquierdo el más frecuente. Comúnmente se acompaña de otras lesiones graves asociadas con mayor mortalidad y morbilidad. Las mejoras recientes de los dispositivos radiológicos han facilitado el diagnóstico; sin embargo, la ausencia de un diagnóstico certero es común, ya que la ruptura diafragmática a menudo es asintomática u obviada. Presentamos un caso de ruptura diafragmática traumática derecha, con el objetivo de destacar la dificultad para realizar el diagnóstico oportunamente y promover la sospecha diagnóstica de esta entidad en pacientes politraumatizados, ya que es frecuente que no se realice la búsqueda intencionada de esta lesión y el retraso diagnóstico puede llevar a mayores complicaciones.

\section{PRESENTACIÓN DE CASO}

Masculino de 47 años de edad acude al servicio de urgencias por presentar traumatismo contuso costal derecho al caer de una motocicleta a baja velocidad. Refiere dolor costal derecho de intensidad 10/10 en la escala visual análoga (EVA) y disnea en reposo.

\section{MATERIAL Y MÉTODOS}

Se aplica protocolo ATLS (Advanced Trauma Life Support) y a la exploración física se evidencian respiraciones superficiales y uso de músculos accesorios. A la auscultación, campos pulmonares hipoventilados de manera generalizada, predominio derecho, con dolor costal a la palpación superficial bilateral. Se administra $\mathrm{O}_{2}$ suplementario, manteniendo una $\mathrm{SpO}_{2}$ 90\%. Se realiza ultrasonido FAST; negativo para líquido libre en cavidad abdominal.

Estudios de laboratorio normales, exceptuando leucocitos: $14.2 \mathrm{mil} / \mathrm{mm}^{3}$, CPK 2,948 $\mu \mathrm{mol} / \mathrm{L}$. Se realiza radiografía de tórax (Figura 1) y tomografía computarizada (TC) toraco-abdominal (Figura 2 a 4) observando neumotórax derecho de $30 \%$, fracturas costales bilaterales de segunda a la séptima. En ambos estudios no se evidencia la presencia de hernia diafragmática.

Se coloca sonda pleural en hemitórax derecho para drenaje de hemoneumotórax. Posteriormente, se realiza toracotomía bilateral con osteosíntesis de fracturas costales sin incidentes. En el posoperatorio, el paciente cursa con datos de dificultad respiratoria persistente e hipoxemia refractaria al uso de oxígeno suplementario, por lo cual se le realiza radiografía de tórax, la cual muestra derrame pleural loculado en hemitórax derecho, resolviéndose con una minitoracotomía derecha con adherenciolisis y drenaje de $400 \mathrm{~mL}$ de líquido pleural hemático y colocación de sonda pleural. El paciente evoluciona con mejoría respiratoria intermitente, sin mejoría en los hallazgos radiográficos subsecuentes, pero sin deterioro significativo en la evolución, por lo que se decide no realizar un nuevo estudio tomográfico. Debido a la ausencia de una esperada mejoría clínica, se decide realizar a los 10 días toracotomía derecha videoasistida encontrando derrame pleural loculado además de un defecto diafragmático posterolateral de 20 $\mathrm{cm}$ con integridad de órganos abdominales.

Se realiza afrontamiento parcial de defecto diafragmático con material no absorbible (polipropileno) con sutura continua por toracoscopía. Posteriormente se ingresa a cavidad abdominal por medio de laparoscopía colocando trocares abdominales (dos de $5 \mathrm{~mm}$ y uno de $10 \mathrm{~mm}$ ), colocando material protésico en el defecto utilizando malla polipropileno de $30 \times 30 \mathrm{~cm}$, utilizando un dispositivo de fijación en toda su extensión.

El paciente evoluciona satisfactoriamente y es dado de alta.

\section{DISCUSIÓN}

La ruptura diafragmática puede ser producida por un trauma contuso o por lesiones penetrantes. De los pacientes con trauma contuso, principalmente víctimas de accidentes automovilísticos o caídas de altura, 0.7-8\% presenta lesiones diafragmáticas; mientras que hasta $15 \%$ se observan en los pacientes con lesiones penetrantes. ${ }^{1,2}$ De $65-75 \%$ de las lesiones ocurren del lado izquierdo, ${ }^{1,2}$

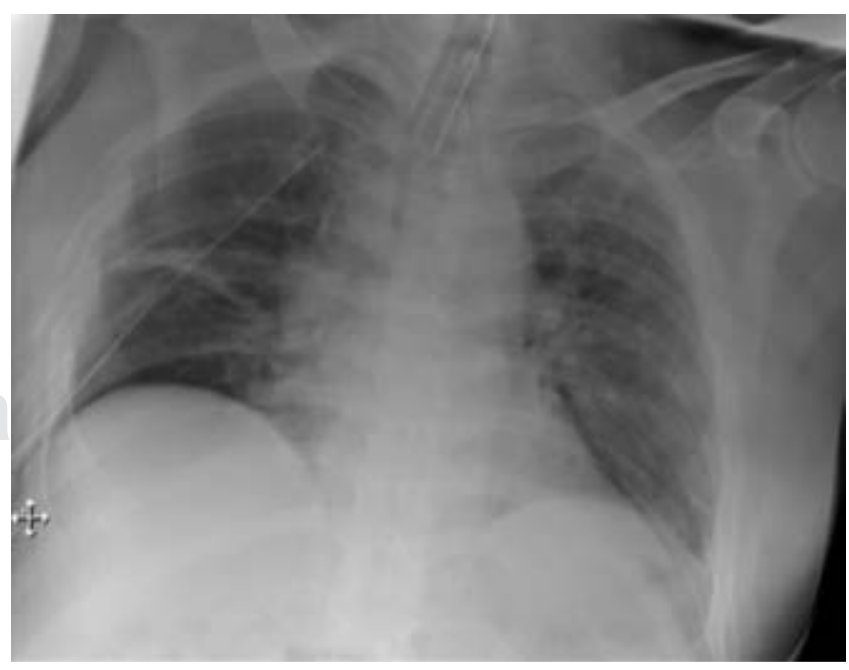

Figura 1: Radiografía de tórax con elevación del hemidiafragma derecho. 


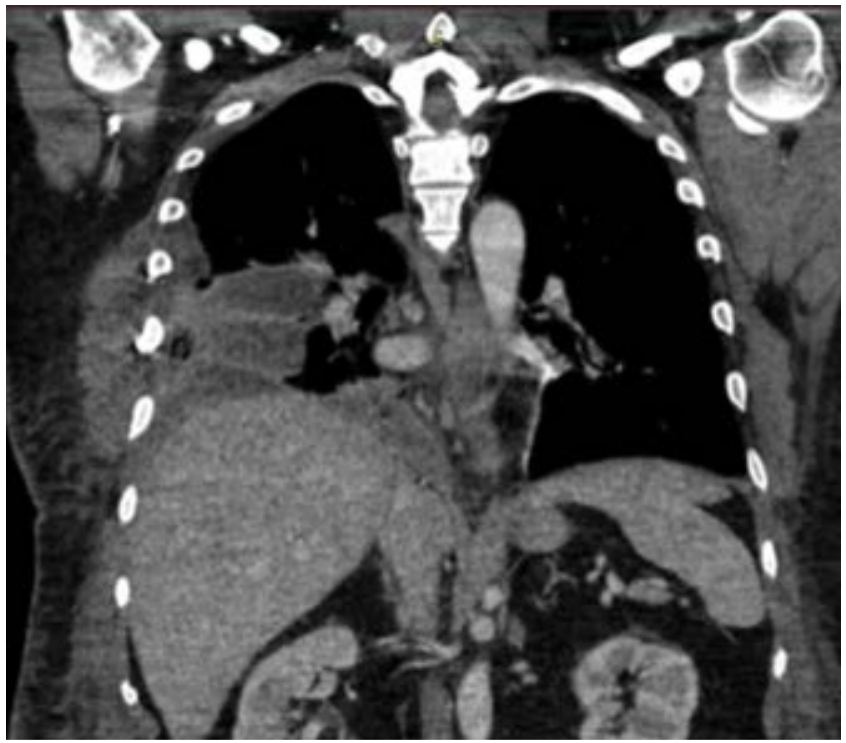

Figura 2: Tomografía de tórax y abdomen con la presencia de vísceras abdominales herniadas a través del diafragma.

ya que el hígado actúa como protector derecho, además de que la mitad izquierda del diafragma se debilita por la presencia del orificio hiatal y el triángulo lumbocostal. ${ }^{2}$ Únicamente $13 \%$ de las lesiones son del lado derecho, con una proporción de 25:1 y $2 \%$ cursa con lesión bilateral. ${ }^{1,3}$

La fisiopatología de una ruptura diafragmática traumática proviene de una elevación súbita de presión intraabdominal por compresión de alta energía o secundaria a trauma abdominal, lo que puede conducir a la ruptura localizada en la parte posterior del diafragma. ${ }^{4}$ Otro mecanismo que puede causar la ruptura se da cuando el impacto es lateral y hay fracturas costales en las cuales se rompen las fijaciones periféricas del diafragma. ${ }^{2}$

Las vísceras abdominales, principalmente estómago e intestino delgado, se pueden herniar a través del defecto diafragmático, llevando así a una insuficiencia respiratoria aguda.

Las lesiones asociadas a una ruptura diafragmática, tales como el traumatismo craneoencefálico, fungen como predictor de mortalidad. ${ }^{4}$ Es raro que el trauma diafragmático se presente aislado, las lesiones más frecuentemente asociadas incluyen lesiones intratorácicas, intraabdominales, fracturas pélvicas, de huesos largos y/o costales. La elevada frecuencia de lesiones asociadas (52-100\%) puede distraer de la lesión diafragmática. ${ }^{5,6}$

Una posibilidad a considerar es el momento de la inserción de una sonda endopleural, la cual puede causar lesión diafragmática, como ha ocurrido en algunos casos reportados en la literatura hasta el momento.

\section{Diagnóstico}

Se debe tener un alto nivel de sospecha clínica, ya que los hallazgos por imagen frecuentemente son poco específicos. El diagnóstico de la ruptura diafragmática puede obviarse hasta en $66 \%$ de los pacientes, dada la dificultad para el mismo al asociarse a otras lesiones diafragmáticas. ${ }^{7}$ La prevalencia de signos positivos en radiografía de tórax varía entre $3-89 \%$ de los $\operatorname{casos}^{2}$ y puede mostrar nivel hidroaéreo intratorácico o la localización intratorácica de la sonda nasogástrica. La elevación de una cúpula diafragmática en ausencia de atelectasias homolaterales y el contorno borroso del diafragma son datos inespecíficos y son difíciles de interpretar en el paciente traumatizado. ${ }^{2}$ La radiografía de tórax conduce al diagnóstico únicamente en $62 \%$ de los pacientes.

En el paciente traumatizado que se encuentra estable, la TC con contraste intravenoso es el estudio de referencia para el diagnóstico y planeación quirúrgica, con una sensibilidad de $14-71 \%$ y especificidad de $76-99 \%{ }^{2}$, y aumenta si se utiliza la TC helicoidal con reconstrucción en 3D (sensibilidad 82-87\%, especificidad $72-99 \%$ ). ${ }^{8}$ Magu y colaboradores reportan una sensibilidad similar a la radiografía de tórax hasta en $50 \% .{ }^{5}$ Los datos tomográficos más sugestivos se muestran en la Tabla 1.2,8

Se han descrito falsos positivos relacionados con defectos posterolaterales en $6 \%$ de los casos correspondientes a una hernia congénita asintomática de Bochdalek. ${ }^{2}$

La TC puede no ser decisiva para el diagnóstico de lesiones diafragmáticas. La elevación aislada de los órganos

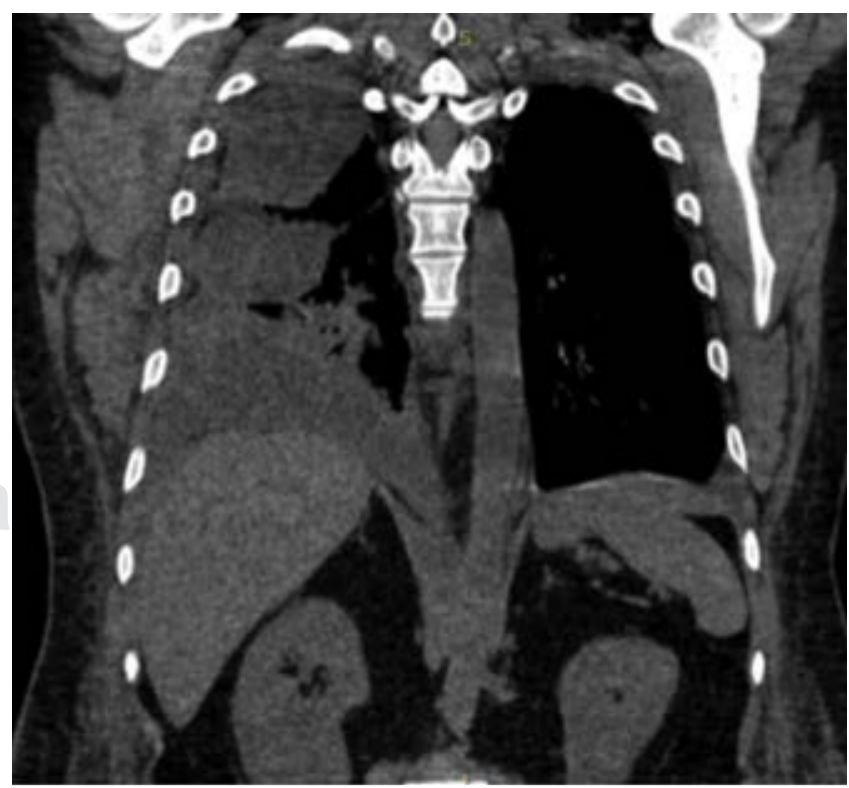

Figura 3: Tomografía de tórax y abdomen con signo de joroba. 


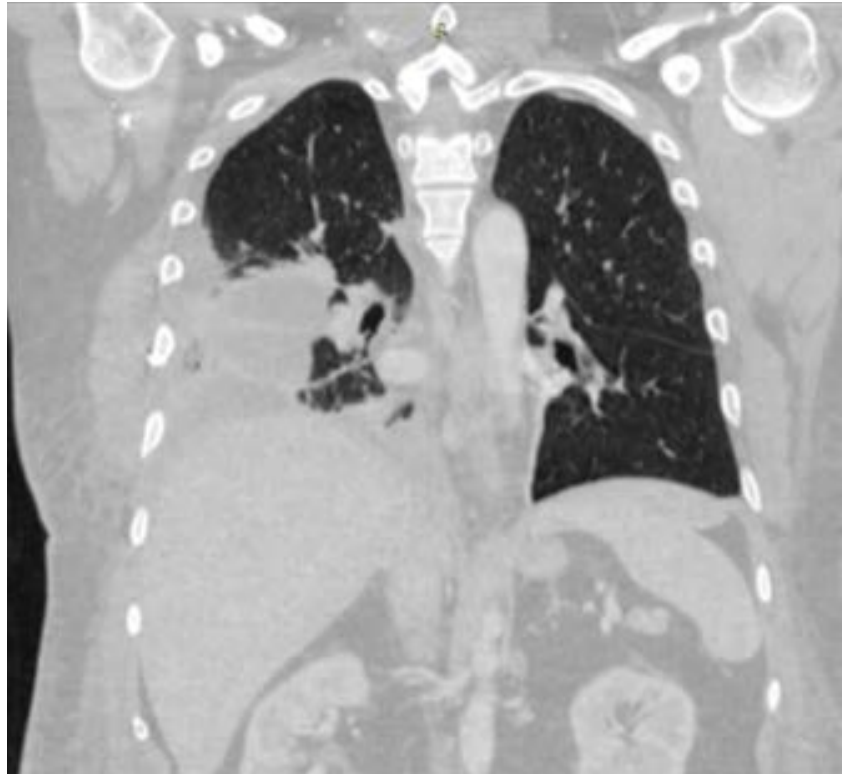

Figura 4: Tomografía de tórax y abdomen con signo del collar.

abdominales y su presencia por encima del diafragma secundaria al defecto de continuidad diafragmática es un síntoma no específico que también se observa en otras entidades nosocomiales como la parálisis del nervio frénico. ${ }^{9}$

\section{Tratamiento}

Se debe realizar la cirugía en el momento del diagnóstico. La reparación primaria del diafragma se puede lograr en agudo debido a la flexibilidad del mismo. Se han descrito técnicas abiertas como la laparotomía y la toracotomía, o enfoques de mínimo acceso como la laparoscopía y la toracoscopía. Se ha demostrado que estas últimas son métodos seguros y efectivos para la evaluación y el tratamiento de las lesiones diafragmáticas agudas. La toracotomía está indicada en casos de hernia diafragmática tardía, lesiones aisladas del diafragma derecho y en casos de sospecha de lesión intratorácica. ${ }^{3}$ La toracoscopía podría ser una opción de tratamiento en casos de ruptura diafragmática unilateral y en pacientes sin otras lesiones intraabdominales asociadas. ${ }^{4}$ Sin embargo, la mayoría de los autores prefieren un abordaje transabdominal, ya que permite la evaluación y el tratamiento de las lesiones primarias y las asociadas como lo hicimos en este caso. ${ }^{4}$

La laparotomía es el abordaje más utilizado en el tratamiento de emergencia del diafragma roto, y se ha demostrado que la ruptura diafragmática se repara con éxito en la mayoría de los casos. Sin embargo, en las últimas dos décadas, se ha agregado una técnica de cirugía de mínimo acceso para reparar el defecto diafragmático, y sus beneficios como la recuperación temprana, buena visión quirúrgica, la reducción del trauma quirúrgico y de la estancia hospitalaria están bien descritos. ${ }^{3,4}$ En nuestra opinión, si se dispone de la experiencia quirúrgica, primero se debe considerar un abordaje laparoscópico, especialmente en un paciente obeso sin otras lesiones asociadas. La laparoscopía diagnóstica puede excluir otras lesiones intraabdominales, evitando así la laparotomía exploratoria innecesaria de la línea media y minimizando el procedimiento para la exploración local de heridas, así como la reparación anatómica en capas. Sin embargo, la laparotomía es la mejor opción en caso de inestabilidad hemodinámica, ya que el desgarro diafragmático rara vez es la causa de un sangrado significativo y de la presencia de lesiones intraabdominales concomitantes como órganos viscerales o lesiones intestinales. En el contexto de lesiones de alta velocidad con una pérdida sustancial de tejido, se debe evitar el uso de una malla hasta lograr una mayor recuperación y estabilidad. ${ }^{4}$

La reparación con malla no se recomienda para pacientes con lesiones asociadas a vísceras huecas u órganos

\section{Tabla 1: Signos tomográficos de lesión diafragmática.}

Signos directos

Discontinuidad diafragmática

Diafragma colgante (el borde roto del diafragma forma una estructura curvilínea con tejidos blandos en un ángulo recto con la pared torácica)

Ausencia de identificación del diafragma

\section{Signos indirectos}

Migración intratorácica de órganos abdominales

Constricción hepática (signo de joroba)

Área lineal de hipoatenuación (signo de la banda)

Signo de vísceras dependientes (contacto de un órgano intraabdominal con la pleura posterior en ausencia de la barrera diafragmática)

Constricción de la masa herniada en el punto de lesión diafragmática (signo del collar) 
sólidos, ya que tales lesiones son fuente de infección. Se recomienda una malla biológica como alternativa a la malla sintética, debido a sus propiedades inherentes de poder incorporarse a los tejidos circundantes con menor riesgo de infección, adhesión, erosión, extrusión y rechazo en comparación con una malla sintética. La reparación fascial primaria dará como resultado una aproximación bajo tensión, lo que puede provocar necrosis y una alta tasa de recurrencia de la hernia. Una reparación primaria con el uso de malla sintética como un injerto subyacente detrás de los músculos con una superposición de al menos $3 \mathrm{~cm}$ es lo ideal, ya que se ha demostrado que está asociada con una tasa de recurrencia más baja que las técnicas de recubrimiento y colocación. Se puede considerar una reparación retrasada después de seis a ocho semanas; sin embargo, se deben considerar riesgos como la encarcelación y el estrangulamiento de los órganos intraabdominales; implica múltiples cirugías, recuperación prolongada y morbilidad adicional en comparación con una reparación de una sola etapa. ${ }^{4}$

La mortalidad estrictamente asociada con la lesión diafragmática es mínima, normalmente está relacionada con las lesiones acompañantes y ésta varía entre $4.3-37 \% .{ }^{1,3}$ Por lo anterior, se enfatiza en la necesidad del reconocimiento temprano y la reparación quirúrgica, ya que un retraso en el diagnóstico puede aumentar la mortalidad hasta en $30 \%$, siendo las principales causas de muerte: choque, falla orgánica múltiple y lesiones craneoencefálicas. ${ }^{3,10}$

\section{CONCLUSIONES}

La ruptura diafragmática secundaria a trauma contuso toracoabdominal puede conducir a una importante morbilidad y mortalidad. El objetivo fue presentar un caso con una elevada dificultad diagnóstica, ya que si no se sospecha el diagnóstico, será difícil acertarlo. Este caso en especial nos alienta a tener siempre presente la posibilidad de que ante un traumatismo cerrado por accidente automovilístico la probabilidad de presentar una hernia diafragmática es muy elevada. Los estudios de imagen siempre nos van a orientar en el diagnóstico, así como en el tratamiento, pero lo más importante es siempre tener en mente este diagnóstico para buscar intencionadamente una hernia diafragmática y de esta manera lograr un tratamiento quirúrgico adecuado.

\section{REFERENCIAS}

1. Porojan VA, David OI, Coman IS, Coman EV, Draghici DA, Popescu C et al. Traumatic diaphragmatic lesions - considerations over a series of 15 consecutive cases. Chirurgia (Bucur). 2019; 114: 73-82.

2. Mancini A, Duramé A, Barbois S, Abba J, Ageron FX, Arvieux C. Relevance of early CT scan diagnosis of blunt diaphragmatic injury: a retrospective analysis from the Northern French Alps Emergency Network. J Visc Surg. 2019; 156: 3-9.

3. Vilallonga R, Pastor V, Alvarez L, Charco R, Armengol M, Navarro S. Right-sided diaphragmatic rupture after blunt trauma. An unusual entity. World J Emerg Surg. 2011; 6: 3.

4. Lee JY, Sul YH, Ye JB, Ko SJ, Choi JH, Kim JS. Right-sided diaphragmatic rupture in a poly traumatized patient. Ann Surg Treat Res. 2018; 94: 342-345.

5. Magu S, Agarwal S, Singla S. Computed tomography in the evaluation of diaphragmatic hernia following blunt trauma. Indian J Surg. 2012; 74: 288-293.

6. Siow SL, Wong CM, Hardin M, Sohail M. Successful laparoscopic management of combined traumatic diaphragmatic rupture and abdominal wall hernia: a case report. J Med Case Rep. 2016; 10: 11.

7. Mehrotra AK, Feroz A, Dawar S, Kumar P, Singh A, Khublani TK. Diaphragmatic rupture precipitated by intercostal chest tube drainage in a patient of blunt thoraco-abdominal trauma. Lung India. 2016; 33: 85-87.

8. Berríos Bárcena J, Hinojosa Velazco O, Flores Revilla E, Mamani Chirinos L. Ruptura diafragmática traumática: presentación de un caso. Rev Colomb Radiol. 2015; 26: 4283-4288.

9. Gmachowska A, Pacho R, Anysz-Grodzicka A, Bakoń L, Gorycka M, Jakuczun W et al. The Role of computed tomography in the diagnostics of diaphragmatic injury after blunt thoraco-abdominal trauma. Pol J Radiol. 2016; 81: 522-528.

10. Zhao L, Han Z, Liu H, Zhang Z, Li S. Delayed traumatic diaphragmatic rupture: diagnosis and surgical treatment. J Thorac Dis. 2019; 11: 2774-2777. 